\title{
Time reversal three-dimensional imaging using single-cycle terahertz pulses
}

\author{
T. Buma ${ }^{a)}$ and T. B. Norris \\ Center for Ultrafast Optical Science, University of Michigan, Ann Arbor, Michigan 48109-2099
}

(Received 25 September 2003; accepted 21 October 2003)

\begin{abstract}
We demonstrate three-dimensional imaging using single-cycle terahertz electromagnetic pulses. Reflection-mode imaging is performed with a photoconductive transmitter and receiver and a reconstruction algorithm based on time reversal. A two-dimensional array is synthesized from ten concentric ring annular arrays with numerical apertures ranging from 0.27 to 0.43 . The system clearly distinguishes image planes separated by $1.5 \mathrm{~mm}$ and achieves a $-6 \mathrm{~dB}$ lateral resolution of $1.1 \mathrm{~mm}$. In terms of the illuminating terahertz power spectrum, the lateral resolution is $38 \%$ and $81 \%$ of the peak and mean wavelengths, respectively. (C) 2004 American Institute of Physics.

[DOI: $10.1063 / 1.1686896]$
\end{abstract}

There is considerable interest in using single-cycle terahertz electromagnetic pulses for imaging. ${ }^{1-6}$ Terahertz waves can penetrate a variety of optically opaque plastics and ceramics. The large bandwidths and submillimeter wavelengths are attractive for spectroscopic imaging. A promising imaging technique is time reversal imaging, ${ }^{1,2}$ which has successfully reconstructed one-dimensional and two-dimensional (2D) objects. In this letter we extend this technique to threedimensional (3D) imaging.

Time reversal imaging is a coherent imaging technique. A transmitted pulse is incident on the object of interest, where the scattered fields are detected by an array of receivers. An image point is reconstructed in a two-step process. First, the time delays are computed for a terahertz pulse to propagate from the transmitter to the image point, and then from the image point to each receiver. Second, the received signal samples corresponding to the computed time delays are summed together to produce the amplitude of the image point. The propagation distances also determine the amplitude weighting of each sample during the summation. It must be emphasized that all the image points are reconstructed using the same received data. Each image point requires a different set of time delays and therefore a different summation of the receiver signals. This "delay-sum" algorithm, commonly used in medical ultrasound imaging, ${ }^{7}$ is equivalent to backpropagating the received signals using the time reversed Huygens-Fresnel diffraction integral. ${ }^{1,2}$

We note that time reversal imaging is similar to Kirchhoff migration ${ }^{5}$ and inverse synthetic aperture $\operatorname{radar}^{6}$ (ISAR). All three techniques coherently sum signals based on propagation distances between the transmitter, object, and receivers. Time reversal and Kirchhoff migration process the signals in the time domain, while ISAR processes signals in the frequency domain. ISAR assumes the object lies in the far field of the synthetic aperture in order to use the fast Fourier transform (FFT) for efficient image reconstruction. ${ }^{8}$ Kirchhoff migration is valid in the near field of the synthetic aperture but neglects the amplitude decay of the diffracting

a)Electronic mail: takbuma@eecs.umich.edu waves. Time reversal makes no approximations to the diffracting waves. Although this increases computational time, rigorous treatment of terahertz diffraction is necessary to achieve our ultimate goal of 3D time-domain diffraction tomography. ${ }^{9}$

The imaging setup is shown in Fig. 1(a). The heart of the system consists of the fiber-coupled photoconductive transmitter and receiver modules. ${ }^{10}$ The fiber-coupling permits arbitrary placement of the modules for synthetic aperture imaging. In contrast to the transmission mode used in previous time reversal imaging, 1,2 the modules are arranged for reflection-mode imaging. The transmitter and receiver are located 142 and $102 \mathrm{~mm}$, respectively, from the origin of the imaging system. The off-axis angles of the transmitter and receiver are given by $\theta_{T}$ and $\theta_{R}$, respectively. The object consists of aluminum foil letters spelling "CUOS" attached to a high-density polyethylene substrate. Each letter is approximately $12 \mathrm{~mm}$ tall and $3 \mathrm{~mm}$ wide with a linewidth of $1 \mathrm{~mm}$. 1.5-mm-deep steps machined into the substrate place each letter at a different $z$ coordinate. The object is approximately centered on the $z$ axis. A ring annular array is synthesized by rotating the object about the $z$ axis while the transmitter and receiver remain stationary. ${ }^{1}$ A 90 -element array is synthesized by rotating the object in $4^{\circ}$ increments after every 100 signal averages. The numerical aperture (NA) of the array is determined by $\mathrm{NA}=\sin \theta_{T}-\sin \theta_{R}$.

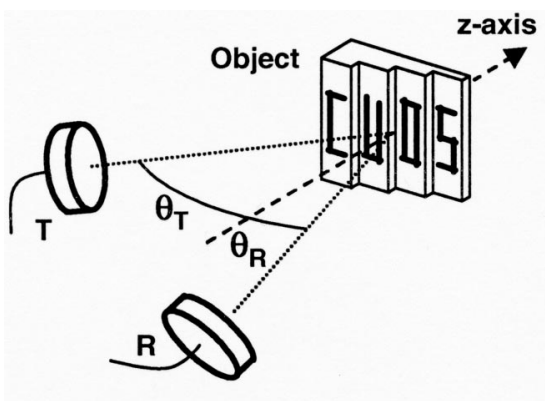

(a)

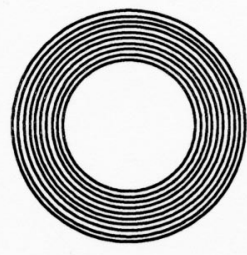

(b)
FIG. 1. Experimental setup for time reversal imaging: (a) reflection imaging configuration and (b) annular synthetic aperture. The transmitter and receiver are labeled as " $T$ " and " $R$," respectively. 


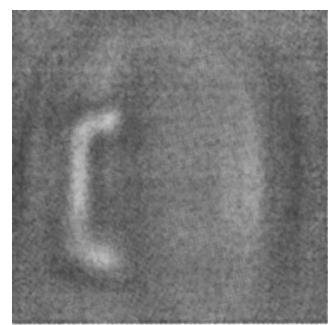

(a)

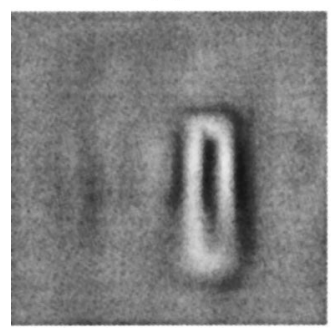

(c)

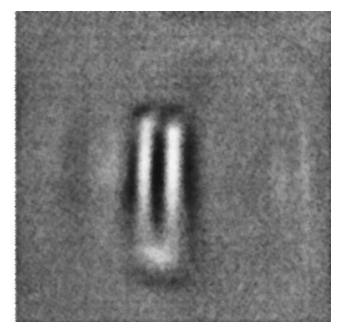

(b)

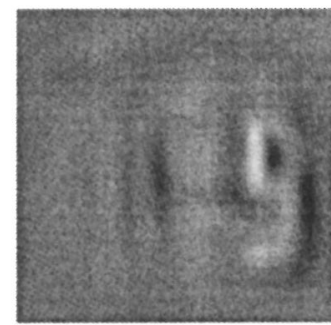

(d)

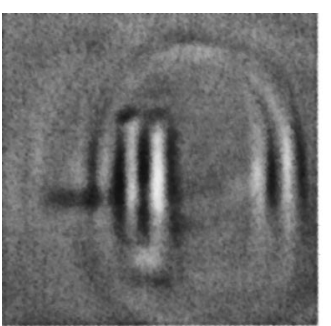

(a)

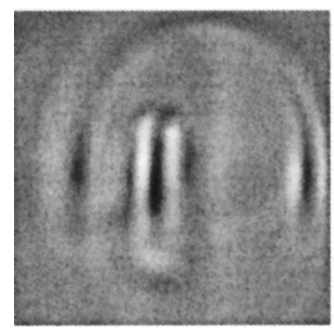

(c)

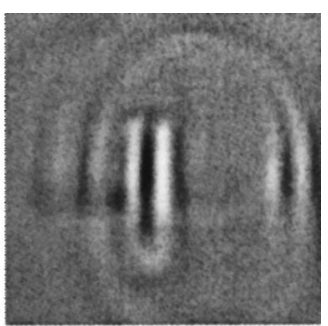

(b)

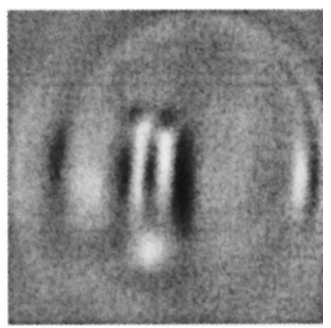

(d)
FIG. 2. Reconstructed image planes for (a) "C," (b) "U," (c) “O," and (d) "S."

Changing $\theta_{R}$ after each full rotation of the object produces a set of concentric ring annular arrays. While maintaining $\theta_{T}=26.5^{\circ}$, ten concentric rings were synthesized by varying $\theta_{R}$ from 1 to $10^{\circ}$ in $1^{\circ}$ increments. As shown in Fig. 1 (b), the multiple rings combine to form an annular imaging aperture with an "inner" NA of 0.27 and an "outer" NA of 0.43 . A reconstructed image is a weighted sum of the images reconstructed from each ring aperture. The weighting is based on approximating the area of the annulus by summing the areas of each ring. Since the rings are distributed along a sphere, the area of a ring is proportional to its NA.

The reconstructed image planes containing the letters "C," "U," "O," and "S" are shown in Figs. 2(a)-2(d), respectively. Each image covers a $25 \times 25 \mathrm{~mm}$ field of view. All images are displayed over the same linear gray scale where white (black) represents positive (negative) field amplitude and mid-gray corresponds to zero. Each letter is clearly reconstructed, demonstrating the ability to distinguish image planes along the $z$ axis. The fainter " $\mathrm{C}$ " and " $\mathrm{S}$ " are due to the limited spot size of the Gaussian-shaped illuminating terahertz beam. Line-outs taken along Figs. 2(a)-2(d) reveal $-6 \mathrm{~dB}$ widths of $1.3,0.8,0.9$, and $1.2 \mathrm{~mm}$, respectively. Reconstructing image points farther away from the origin is more sensitive to slight errors in alignment of the transmitter and receiver modules. The average lateral resolution is $1.1 \mathrm{~mm}$, corresponding to $38 \%$ and $81 \%$ of the peak and mean wavelengths, respectively, of the illuminating terahertz power spectrum.

In every image of Fig. 2, faint patches of white and black are visible in regions away from a letter. These artifacts are shown more vividly by the images reconstructed from a single ring rather than the weighted sum of all the rings. Figures $3(\mathrm{a})-3(\mathrm{~d})$ show reconstructed images of the letter " $U$ " from single rings with $N A=0.43,0.38,0.32$, and 0.27 , respectively. The most striking features are the bright arcs sweeping across the images. When combining images from multiple rings, these artifacts fail to reinforce each other since their positions are different in each image. In contrast, the reconstructed letters reinforce each other since
FIG. 3. Reconstructed images of the letter "U" using a single ring with (a) $\mathrm{NA}=0.43$, (b) $\mathrm{NA}=0.38$, (c) $\mathrm{NA}=0.32$, and (d) $\mathrm{NA}=0.27$.

their positions in the images remain the same. As a result, the annular array images in Fig. 2 have weaker artifacts than the single-ring images of Fig. 3.

The origin of these artifacts can be understood by analyzing the point spread function (PSF) of a single ring array. The analysis is simplified by assuming the array as continuously sampled around its circumference. The paraxial approximation is applied to the time-reversed HuygensFresnel diffraction integral, ${ }^{1}$ which is commonly made in the analysis of ultrasonic transducer arrays. ${ }^{11}$ A point object is placed at the origin of the system. Expressed in cylindrical coordinates, the reconstructed image field is given by

$$
\begin{aligned}
u(r, z)= & \int_{-\infty}^{\infty} \frac{d \omega}{2 \pi} \kappa_{0} \omega^{2} u_{\mathrm{in}}(\omega) J_{0}(\omega \mathrm{NA} r / c) \\
& \times \exp (-i \omega z \zeta / c),
\end{aligned}
$$

where $\zeta=\cos \theta_{T}+\cos \theta_{R}$ and $c$ is the speed of light in air. The term $\kappa_{0}$ is a geometrical factor depending on the synthetic array dimensions, while $u_{\text {in }}(\omega)$ is the frequency spectrum of the terahertz field incident on the point target. When $r$ is much larger than the peak wavelength of the terahertz spectrum, the Bessel function $J_{0}$ in Eq. (1) can be replaced by its large argument expansion to give

$$
\begin{aligned}
u(r, z)= & \int_{-\infty}^{\infty} \frac{d \omega}{2 \pi} \kappa_{0} \omega^{2} u_{\mathrm{in}}(\omega) \frac{\cos (\omega \mathrm{NA} r / c-\pi / 4)}{\sqrt{2 \pi \omega \mathrm{NA} r / c}} \\
& \times \exp (-i \omega z \zeta / c) .
\end{aligned}
$$

Applying the principle of stationary phase, the major contribution to the integral in Eq. (2) occurs when $r=|z| \zeta /$ NA. This produces a ring-shaped halo in the reconstructed image of a plane located a distance $|z|$ from the point object. The halo radius varies with the numerical aperture of the array.

We performed simulations to visualize this halo artifact. The model consists of a 0.47 NA ring annular array imaging a point scatterer at the origin. All gray scale images cover a $12.5 \times 12.5 \mathrm{~mm}$ area. The reconstructed image of the $x-y$ plane containing the point object is shown in Fig. 4(a). This 


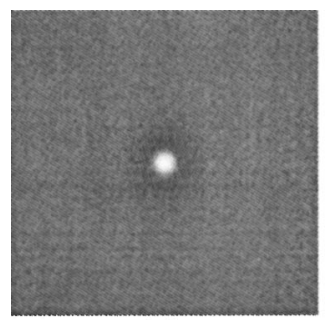

(a)

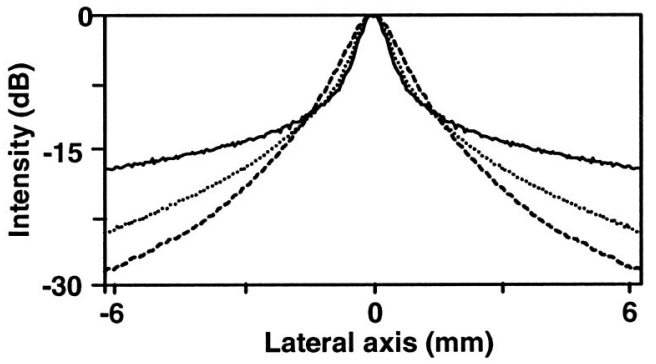

(c)

FIG. 4. Simulated point spread function (PSF) along (a) $x-y$ plane at $z$ $=0 \mathrm{~mm}$ and (b) $x-y$ plane at $\mathrm{z}=-1 \mathrm{~mm}$. The image intensity is computed along the dashed line. (c) Integrated intensity plot for a single ring aperture (solid curve). The plots for 10 rings (dotted curve) and 26 rings (dashed curve) show how more highly filled 2D apertures suppress artifacts.

is what one intuitively expects for the lateral PSF. The reconstructed image of the $x-y$ plane $1 \mathrm{~mm}$ in front of the point object $(z=-1 \mathrm{~mm})$ is shown in Fig. 4(b). The circular feature is the previously described halo. This helps explain the artifacts in Fig. 2; the bright arcs are from letters in other object planes. In other words, the reconstructed image is corrupted by scattered waves from out-of-plane objects. Consequently, 3D imaging is not simply a matter of using a singlering annular array ${ }^{1}$ to reconstruct multiple depths.

The strength of the halo artifact is measured by computing the image intensity along the dashed line in Fig. 4(b). This is repeated for all image planes distributed within $-6.25<z<6.25 \mathrm{~mm}$. The intensity function is then integrated over $z$ and displayed by the solid curve in Fig. 4(c). The central lobe reveals the lateral resolution of the system, while the slowly decaying pedestal indicates the strength of artifacts. The dotted curve shows the simulated beam plot of the ten-ring aperture used in the experiment. The much lower pedestal reveals that artifacts are indeed suppressed by using a more highly filled 2D aperture. An even lower pedestal is shown by the dashed curve, which simulates a completely filled disk aperture synthesized from 26 rings. The central lobe is slightly wider since the smaller diameter rings contribute lower spatial frequencies. However, the dramatic suppression in artifacts far outweighs the slight broadening of the lateral PSF.

We have demonstrated time reversal 3D imaging using single-cycle terahertz electromagnetic pulses. The system clearly distinguishes image planes separated by $1.5 \mathrm{~mm}$ and achieves a $-6 \mathrm{~dB}$ lateral resolution $38 \%$ and $81 \%$ of the peak and mean terahertz wavelengths, respectively. 3D reconstruction artifacts are excessively high using a single-ring annular array. As a result, a more highly filled 2D aperture is necessary to suppress the artifacts. Future work involves imaging with a fully populated disk array, which should produce the highest image quality. A combination of reflectionmode and transmission-mode imaging will also be done to perform 3D time-domain diffraction tomography.

${ }^{1}$ A. B. Ruffin, J. V. Rudd, J. Decker, L. Sanchez-Palencia, L. Lehors, J. F. Whitaker, and T. B. Norris, IEEE J. Quantum Electron. 38, 1110 (2002).

${ }^{2}$ A. B. Ruffin, J. Decker, L. Sanchez-Palencia, L. Lehors, J. F. Whitaker, T. B. Norris, and J. V. Rudd, Opt. Lett. 26, 681 (2001).

${ }^{3}$ B. Ferguson, S. Wang, D. Gray, D. Abbot, and X. C. Zhang, Opt. Lett. 27, 1312 (2002).

${ }^{4}$ J. O'Hara and D. Grischkowsky, Opt. Lett. 27, 1070 (2002).

${ }^{5}$ T. D. Dorney, J. L. Johnson, J. V. Rudd, R. G. Baraniuk, W. W. Symes, and D. M. Mittleman, Opt. Lett. 26, 1513 (2001).

${ }^{6}$ K. McClatchey, M. T. Reiten, and R. A. Cheville, Appl. Phys. Lett. 79, 4485 (2001).

${ }^{7}$ A. Macovski, Medical Imaging Systems (Prentice-Hall, Englewood Cliffs, NJ, 1983).

${ }^{8}$ D. L. Mensa, High Resolution Radar Cross-Section Imaging (Artech House, Norwood, MA, 1991).

${ }^{9}$ T. Melamed, Y. Ehrlich, and E. Heyman, Inverse Probl. 12, 977 (1996).

${ }^{10}$ Picometrix, Inc., Ann Arbor, MI.

${ }^{11}$ S. J. Norton, J. Acoust. Soc. Am. 92, 3202 (1992). 